\title{
TRUNCATION-TYPE METHODS AND BÄCKLUND TRANSFORMATIONS FOR ORDINARY DIFFERENTIAL EQUATIONS: THE THIRD AND FIFTH PAINLEVÉ EQUATIONS
}

\author{
P. R. GORDOA \\ Area de Fisica Teórica, Facultad de Ciencias, Edificio de Fisica, Universidad de Salamanca, \\ 37008 Salamanca, Spain \\ e-mail:prg@sonia.usal.es

\section{N. JOSHI} \\ Department of Pure Mathematics, University of Adelaide, Adelaide, Australia 5005 \\ e-mail: nalini.joshi@adelaide.edu.au \\ and A. PICKERING \\ Area de Fisica Teórica, Facultad de Ciencias, Edificio de Fisica, Universidad de Salamanca, \\ 37008 Salamanca, Spain \\ e-mail: andrew@sonia.usal.es
}

(Received 29 December, 1999; revised 15 June, 2000)

\begin{abstract}
In a recent paper we presented a truncation-type method of deriving Bäcklund transformations for ordinary differential equations. This method is based on a consideration of truncation as a mapping that preserves the locations of a natural subset of the movable poles that the equation possesses. Here we apply this approach to the third and fifth Painlevé equations. For the third Painlevé equation we are able to obtain all fundamental Bäcklund transformations for the case where the parameters satisfy $\gamma \delta \neq 0$. For the fifth Painleve equation our approach yields what appears to be all known Bäcklund transformations.
\end{abstract}

2000 Mathematics Subject Classification. 34M55, 33E17.

1. Introduction. In a recent paper [1] we introduced a new approach to finding Bäcklund transformations for ordinary differential equations (ODEs). This truncation-type method constituted an extension to ODEs of an approach that had been successfully developed for partial differential equations (PDEs) [2]. The main idea in [1] is to consider truncation as a mapping that preserves the locations of a natural subset of movable singularities. The generic solution of each of the Painleve equations (except $P_{I}$ ) has pairs of movable simple poles with leading order coefficients of opposite sign. Thus the set of all poles of a solution $y(x)$ decomposes into the union of two nonintersecting subsets $\mathcal{P}_{+}$and $\mathcal{P}_{-}$, where $\mathcal{P}_{+}$is the set of poles with positive choice of coefficient and $\mathcal{P}_{-}$is that with negative choice. In what follows we transform a generic solution $y(x)$ of a Painlevé equation to a solution $Q(x)$ of the same equation, but with possibly different parameters, as

$$
y(x)=\rho(x)+Q(x),
$$

where we demand that $\rho(x)$ has poles at the elements of $\mathcal{P}_{+}$and $Q(x)$ has them at $\mathcal{P}_{-}$, or vice versa. We are then able to find Bäcklund transformations through a procedure that relies only on singularity analysis of the transformed equation. 
In [1] we applied this approach to $P_{I I}$ and $P_{I V}$. In addition to obtaining Bäcklund transformations for these equations we also discussed transformations to related ODEs (the ODEs satisfied by $\rho(x)$ ), as well as the application of a "doublesingularity approach." In the present work we apply our approach to $P_{I I I}$ and $P_{V}$, in order to obtain Bäcklund transformations. Transformations to related ODEs and the double-singularity approach for $P_{I I I}$ and $P_{V}$ will be considered elsewhere. Descriptions of Bäcklund transformations for $P_{I I I}$ and $P_{V}$ can be found in [3-9].

2. Bäcklund transformations for $\boldsymbol{P}_{I I I}$. We take the third Painlevé equation in the form

$$
y^{\prime \prime}=\frac{\left(y^{\prime}\right)^{2}}{y}-\frac{y^{\prime}}{x}+\frac{\alpha y^{2}+\beta}{x}+\gamma^{2} y^{3}-\frac{\delta^{2}}{y}
$$

where we have renamed two of the parameters as $\gamma^{2}$ and $-\delta^{2}$ (conventionally labelled as $\gamma$ and $\delta$ respectively). A generic solution $y(x)$ of the third Painlevé equation is transformed to another solution $Q(x)$ of the same equation but with possibly different parameters $a, b, c$ and $d$ as

$$
y(x)=\rho(x)+Q(x) .
$$

The dominant terms in the expression that results from the substitution of equation (3) into (2) are, assuming $\gamma \neq 0$,

$$
\rho \rho^{\prime \prime}-\left(\rho^{\prime}\right)^{2} \approx \gamma^{2} \rho^{4}
$$

which can be integrated to give

$$
\rho^{\prime} \approx \pm \gamma \rho^{2}
$$

Taking first of all the minus sign in this last equation, we write

$$
\rho^{\prime}=-\gamma \rho^{2}+\sigma \rho
$$

Substituting this into the transformed version of equation (2) and looking once again at dominant terms, we get

$$
\sigma(x)=-2 \gamma Q(x)-\frac{\alpha+\gamma}{\gamma x}+\frac{\tau}{\rho} .
$$

Using this in the transformed equation yields a linear equation for $\rho$, which has to be compatible with the Riccati equation

$$
\rho^{\prime}=-\gamma \rho^{2}-\left(2 \gamma Q(x)+\frac{\alpha+\gamma}{\gamma x}\right) \rho+\tau .
$$

The analysis of the resulting compatibility condition depends on whether or not $\tau$ is assumed to depend only on $x$ or if it is allowed to depend also on $Q(x)$. (We do not 
consider here the possible dependence of $\tau$ on $Q^{\prime}(x)$.) Assuming $\tau=\tau(x, Q(x))$ and using the fact that $Q(x)$ satisfies $P_{I I I}$, we obtain a polynomial in $Q^{\prime}(x)$. The coefficient of the highest degree term gives

$$
Q^{2} \tau_{Q Q}-Q \tau_{Q}+\tau=0
$$

whose general solution is given by

$$
\tau=\left[f_{1}(x)+f_{2}(x) \log Q\right] Q,
$$

where $f_{1}(x)$ and $f_{2}(x)$ are functions to be determined. If we now insert this form for $\tau$ in the compatibility condition and set to zero coefficients of the resulting polynomial in $Q, Q^{\prime}$ and $\log Q$, we first obtain

$$
\begin{aligned}
f_{1}(x) & =\frac{a-\alpha}{\gamma x}, \\
f_{2}(x) & =0, \\
c^{2} & =\gamma^{2}, \\
d^{2} & =\delta^{2},
\end{aligned}
$$

which means that the actual form of $\tau$ is

$$
\tau(x, Q)=\frac{a-\alpha}{\gamma x} Q .
$$

Note that if we had taken $\tau$ to be a function of $x$ only, our compatibility condition would have led to $\tau=0$ and thus to $a=\alpha$, and we would only have obtained restricted results. The main difference between the application of our method to $P_{I I I}$, and its previous application to $P_{I I}$ and $P_{I V}$ [1], is that for $P_{I I I}$, allowing $\tau$ to depend on $Q$ leads to more general results, whereas for $P_{I I}$ and $P_{I V}$ it does not.

Inserting the above results into the compatibility condition, we obtain from the coefficient of the next term (in the polynomial in $Q^{\prime}$ ) the parameter shift,

$$
2 \gamma(b-\beta)+b a-\beta \alpha=0 .
$$

We now consider separately the remainder of our compatibility condition for the two cases $b=0$ and $b \neq 0$. We take first the case $b \neq 0$. Solving equation (16) for $a$ and substituting back into the compatibility condition gives the following additional constraints between the parameters,

$$
\begin{array}{r}
(b-\beta)(b+\beta)(\gamma b-\delta \alpha-2 \gamma \delta)(\gamma b+\delta \alpha+2 \gamma \delta)=0, \\
(b-\beta)(b+\beta)(\gamma b-\delta \alpha-2 \gamma \delta)(\gamma b+\delta \alpha+2 \gamma \delta)(2 \gamma \beta+\beta \alpha-\gamma b)=0 .
\end{array}
$$

Taking $b=\beta$ in (17) just leads to the identity $y(x)=Q(x)$. However the remaining factors in (17) lead to nontrivial Bäcklund transformations. Taking $b=-\beta$ leads to the Bäcklund transformation 


$$
\begin{aligned}
\rho= & -2 Q^{2} \frac{A_{1}\left(x, Q, Q^{\prime}\right)}{A_{2}\left(x, Q, Q^{\prime}\right)} \\
A_{1}= & \left(2 \gamma^{2} x+\alpha \gamma x\right) Q^{\prime}+\left(\alpha \gamma^{2} x+2 \gamma^{3} x\right) Q^{2}-\left(6 \gamma^{2}+5 \alpha \gamma+\alpha^{2}\right) Q-\beta \gamma^{2} x, \\
A_{2}= & \gamma^{2} x^{2}\left(Q^{\prime}\right)^{2}+2 \gamma^{3} x^{2} Q^{2} Q^{\prime}-2 \gamma^{2} x Q Q^{\prime}+\gamma^{4} x^{2} Q^{4}-2 \gamma^{3} x Q^{3} \\
& -\left(3 \gamma^{2}+4 \alpha \gamma+\alpha^{2}\right) Q^{2}-2 \beta \gamma^{2} x Q-\delta^{2} \gamma^{2} x^{2}, \\
a= & -\alpha-4 \gamma \\
b= & -\beta \\
c^{2}= & \gamma^{2} \\
d^{2}= & \delta^{2},
\end{aligned}
$$

whereas taking $\gamma b \pm \delta(\alpha+2 \gamma)=0$ (which requires $\delta \neq 0$ ) leads to

$$
\begin{aligned}
\rho & =\mp \frac{(\gamma \beta \pm \delta \alpha \pm 2 \gamma \delta) Q^{2}}{\delta\left[\gamma x Q^{\prime}+\gamma^{2} x Q^{2}+(\alpha+\gamma) Q \pm \delta \gamma x\right]}, \\
a & =-\gamma\left(2 \pm \frac{\beta}{\delta}\right), \\
b & =\mp\left(2 \delta+\frac{\alpha \delta}{\gamma}\right), \\
c^{2} & =\gamma^{2}, \\
d^{2} & =\delta^{2} .
\end{aligned}
$$

Here the choice of sign of $\delta$ arises because of the way we have written this parameter in $P_{I I I}$.

If we now consider the case with $b=0$ and we use this in the remainder of our compatibility condition, we recover the two Bäcklund transformations above (with $b=0)$, and in addition

$$
\begin{aligned}
\rho & =\frac{(a-\alpha) Q^{2}}{\gamma x Q^{\prime}+\gamma^{2} x Q^{2}+(\gamma+\alpha) Q}, \\
b & =\beta=0, \\
c^{2} & =\gamma^{2}, \\
d & =\delta=0 .
\end{aligned}
$$

We note however that in the case $\beta=\delta=0, P_{I I I}$ is explicitly solvable $[\mathbf{4 , 8 , 9 ]}$.

We now consider taking the opposite sign in front of the term in $\rho^{2}$ in (6), i.e.

$$
\rho^{\prime}=\gamma \rho^{2}+\sigma \rho
$$

However the results thus obtained can be written down simply by changing the sign of $\gamma$ in the results obtained above.

Thus far we have assumed $\gamma \neq 0$. However, since the change of variables

$$
y(x)=\frac{1}{m(x)}
$$


transforms $P_{I I I}$ in $y(x)$ into $P_{I I I}$ in $m(x)$ but with new parameters $\tilde{\alpha}=-\beta, \tilde{\beta}=-\alpha$, $\tilde{\gamma}^{2}=\delta^{2}$ and $\tilde{\delta}^{2}=\gamma^{2}$, the case $\gamma=0$ can be dealt with as above provided that $\delta \neq 0$. The remaining case $\gamma=\delta=0$ can be dealt with by another change of variables which maps $P_{I I I}$ with parameters $\alpha, \beta, \gamma=0$ and $\delta=0$ onto $P_{I I I}$ with parameters $\hat{\alpha}=0, \hat{\beta}=0, \hat{\gamma}=2 \alpha$ and $\hat{\delta}=2 \beta$ [9] (and noting that if in addition $\alpha=0$ or $\beta=0$ the original copy of $P_{I I I}$ is in any case explicitly solvable $\left.[\mathbf{4 , 8}, \mathbf{9}]\right)$.

The four Bäcklund transformations obtained by taking (26)-(30) together with the possible change of sign $\gamma \rightarrow-\gamma$ correspond to the four fundamental Bäcklund transformations for $P_{I I I}$ in the case $\gamma \delta \neq 0$ (denoted $T_{i}, i=1,2,3,4$, in [8]; see also [4]). All other known Bäcklund transformations for $P_{I I I}$ in this case $\gamma \delta \neq 0$ can be expressed in terms of these $T_{i}$ together with (36) and simple rescalings [8].

The two Bäcklund transformations obtained by taking (19)-(25) together with the possible change of sign $\gamma \rightarrow-\gamma$ correspond in the case $\delta=0$ (and $\beta \gamma \neq 0$ ) to a second iteration of a Bäcklund transformation given in [9] (see also Theorem 4.1 in [4] for $\gamma=0$, or Transformation $\mathrm{V}$ in [8], for $\alpha \delta \neq 0$ ) combined with (36) and suitable rescalings. In the case $\delta \neq 0$ they correspond to the second iteration of appropriate combinations of (26)-(30) (i.e. of the transformations $T_{i}$ in [8]). However the general formulation of these second iterations presented here, of Bäcklund transformations which are usually treated separately, appears to be new.

We note that consideration of the ODE satisfied by $\rho(x)$ leads to Bäcklund transformations between $P_{I I I}$ and an ODE of second order and second degree, and between $P_{I I I}$ and $P_{V}$, for (19)-(25) and (26)-(30) respectively.

3. Bäcklund transformations for $\boldsymbol{P}_{V}$. We take $P_{V}$ in the form

$$
y^{\prime \prime}=\left(\frac{1}{2 y}+\frac{1}{y-1}\right)\left(y^{\prime}\right)^{2}-\frac{y^{\prime}}{x}+\frac{(y-1)^{2}}{2 x^{2}}\left(\alpha^{2} y-\frac{\beta^{2}}{y}\right)+\frac{\gamma y}{x}-\frac{\delta^{2} y(y+1)}{2(y-1)},
$$

where we have renamed three of the parameters as $\alpha^{2} / 2,-\beta^{2} / 2$ and $-\delta^{2} / 2$ (conventionally labelled as $\alpha, \beta$ and $\delta$ respectively). When applying the approach outlined above to $P_{V}$ in this form, the presence of a resonance at first order after the leading term means that $\sigma$ [in an equation corresponding to (6)] is not determined by a linear algebraic equation. Rather than continuing with the resulting set of equations thus obtained, we make instead the change of variables

$$
y(x)=\frac{m(x)}{m(x)-1}
$$

which gives

$$
\begin{aligned}
m(m-1) x^{2} m^{\prime \prime}= & \left(m-\frac{1}{2}\right) x^{2}\left(m^{\prime}\right)^{2}+m(1-m) x m^{\prime}+\delta^{2} x^{2} m^{5} \\
& -\left(\gamma+\frac{5}{2} x \delta^{2}\right) x m^{4}+2\left(\gamma+\delta^{2} x\right) x m^{3} \\
& +\frac{1}{2}\left(\beta^{2}-\alpha^{2}-\delta^{2} x^{2}-2 \gamma x\right) m^{2}-\beta^{2} m+\frac{1}{2} \beta^{2},
\end{aligned}
$$

in which form no resonance at first order after the leading term occurs. A generic solution $m(x)$ of this last equation is transformed into another solution $Q(x)$ of the 
same equation (39) but with possibly different values of the parameters that we denote by $a, b, c$ and $d$, as

$$
m(x)=\rho(x)+Q(x)
$$

Substitution of (40) into equation (39) gives an equation whose dominant terms near a pole of $\rho$, assuming $\delta \neq 0$, are

$$
\delta^{2} \rho^{5}+\rho\left(\rho^{\prime}\right)^{2} \approx \rho^{2} \rho^{\prime \prime}
$$

which can be integrated to give

$$
\rho^{\prime} \approx \pm \delta \rho^{2}
$$

We consider first the case with the plus sign in this last, and write

$$
\rho^{\prime}=\delta \rho^{2}+\sigma \rho .
$$

Substituting this into the transformed version of equation (39) and looking once again at dominant terms, we get

$$
\sigma(x)=2 \delta Q(x)-\frac{\delta+\gamma+\delta^{2} x}{\delta x}+\frac{\tau}{\rho} .
$$

Using this in the transformed equation we obtain, as we did for the fourth Painlevé equation [1], a quadratic in $\rho$ which has to be compatible with the Riccati equation

$$
\rho^{\prime}=\delta \rho^{2}+\left(2 \delta Q(x)-\frac{\delta+\gamma+\delta^{2} x}{\delta x}\right) \rho+\tau .
$$

Again the analysis of the resulting compatibility condition depends on whether or not $\tau$ is assumed to depend only on $x$, or on both $x$ and $Q(x)$. [Once again, for simplicity, we do not consider here the case where $\tau$ may depend on $Q^{\prime}(x)$.] Assuming $\tau=\tau(x, Q(x))$, and using the fact that $Q$ satisfies $P_{V}$, we obtain a polynomial in $Q^{\prime}$ whose coefficient at highest degree gives the following differential equation for $\tau$,

$$
2 Q\left(2 Q^{3}-4 Q^{2}+3 Q-1\right) \tau_{Q Q}+\left(-4 Q^{3}+6 Q^{2}-1\right) \tau_{Q}+2\left(2 Q^{2}-2 Q-1\right) \tau=0 .
$$

This has general solution

$$
\tau=(2 Q-1)\left[f_{1}(x)+f_{2}(x) \log \left(Q-\frac{1}{2}+\sqrt{Q(Q-1)}\right)\right]+2 f_{2}(x) \sqrt{Q(Q-1)},
$$

where $f_{1}(x)$ and $f_{2}(x)$ are functions to be determined. Inserting this form for $\tau$ into the compatibility condition we next obtain

$$
\begin{aligned}
f_{1}(x) & =\frac{1}{2 \delta x}(c-\gamma), \\
f_{2}(x) & =0, \\
d^{2} & =\delta^{2},
\end{aligned}
$$


which means that $\tau$ in fact takes the form

$$
\tau=\frac{1}{2 \delta x}(c-\gamma)(2 Q-1)
$$

From this last we see that if we had assumed $\tau$ to be a function of $x$ only, then as for $P_{I I I}$ we would only have obtained restricted results. Thus once again we see that allowing $\tau$ to depend on $Q$ leads to more general results.

Using the above results in our compatibility condition, we obtain from the next higher order term the following shift between the parameters,

$$
a^{2}=\frac{\gamma^{2}-c^{2}+2 \delta^{2}\left(\alpha^{2}+\beta^{2}-b^{2}\right)+2 \delta(\gamma-c)}{2 \delta^{2}} .
$$

Using this in our compatibility condition then leads to

$b^{2}=\frac{4 \beta^{2} \delta^{3}+2\left[(c+\gamma) \beta^{2}+(c-\gamma)\left(\alpha^{2}-1\right)\right] \delta^{2}+\left(\gamma^{2}-3 c^{2}+2 c \gamma\right) \delta-c^{3}+c \gamma^{2}}{4 \delta^{2}(c+\delta)}$

where we have assumed that $c+\delta \neq 0$ (the case $c+\delta=0$ has to be considered separately). Substituting back once again then provides the additional condition

$$
\begin{aligned}
(c-\gamma)(c+\gamma+2 \delta)(c+\delta-\delta \alpha-\delta \beta)(c+\delta+\delta \alpha-\delta \beta) & (c+\delta-\delta \alpha+\delta \beta) \\
& \times(c+\delta+\delta \alpha+\delta \beta)=0 .
\end{aligned}
$$

This provides essentially three different cases to consider, since the last three factors are related to the third under changes of sign of $\alpha$ and $\beta$. These three cases are:

$$
\begin{aligned}
& c=\gamma, \\
& c=-\gamma-2 \delta, \\
& c=\delta(\beta+\alpha-1) .
\end{aligned}
$$

The case $c=\gamma$ corresponds to the identity transformation (this is easily seen by substituting this condition into the above expressions for $a^{2}$ and $b^{2}$.) The other two cases lead to two nontrivial Bäcklund transformations, i.e.

$$
\begin{aligned}
\rho= & Q(Q-1) \frac{A_{1}\left(x, Q, Q^{\prime}\right)}{A_{2}\left(x, Q, Q^{\prime}\right)} \\
A_{1}= & -2 \delta x(\delta+\gamma) Q^{\prime}+2 \delta^{2} x(\delta+\gamma) Q^{2}+2\left(\delta^{2}-\delta^{3} x+2 \gamma \delta-\delta^{2} \gamma x+\gamma^{2}\right) Q \\
& +\delta^{2}\left(\beta^{2}-\alpha^{2}-1\right)-2 \gamma \delta-\gamma^{2}, \\
A_{2}= & \delta^{2} x^{2}\left(Q^{\prime}\right)^{2}+2 \delta^{3} x^{2}\left(Q-Q^{2}\right) Q^{\prime}+\delta^{4} x^{2} Q^{4}-2 \delta^{4} x^{2} Q^{3}-\alpha^{2} \delta^{2} \\
& +\left(\delta^{4} x^{2}-\delta^{2}-2 \gamma \delta-\gamma^{2}\right) Q^{2}+\left(\delta^{2}+\alpha^{2} \delta^{2}-\beta^{2} \delta^{2}+2 \gamma \delta+\gamma^{2}\right) Q, \\
a^{2}= & \beta^{2} \\
b^{2}= & \alpha^{2} \\
c= & -\gamma-2 \delta, \\
d^{2}= & \delta^{2},
\end{aligned}
$$


and

$$
\begin{aligned}
\rho & =\frac{2 Q(Q-1)(\alpha \delta+\beta \delta-\gamma-\delta)}{2 \delta x Q^{\prime}-2 \delta^{2} x Q^{2}+2\left(\gamma+\delta+\delta^{2} x\right) Q+\delta(\alpha-\beta-1)-\gamma}, \\
a^{2} & =\left[\frac{\gamma+\delta(\alpha-\beta+1)}{2 \delta}\right]^{2}, \\
b^{2} & =\left[\frac{-\gamma+\delta(\alpha-\beta-1)}{2 \delta}\right]^{2}, \\
c & =\delta(\beta+\alpha-1), \\
d^{2} & =\delta^{2} .
\end{aligned}
$$

We also have of course Bäcklund transformations obtained from the above under combinations of $\alpha \rightarrow-\alpha, \beta \rightarrow-\beta$. As mentioned earlier, we also have our compatibility condition for the case $c+\delta=0$, which needs to be considered separately. However this leads only to restricted cases of the above Bäcklund transformations.

We now consider taking the opposite sign in front of the term in $\rho^{2}$ in (43), i.e.

$$
\rho^{\prime}=-\delta \rho^{2}+\sigma \rho
$$

The results thus obtained are as above with $\delta \rightarrow-\delta$.

In the above we have assumed $\delta \neq 0$. We note that $P_{V}$ in the case $\delta=0$ can be reduced to $P_{I I I}$ with $\gamma \delta \neq 0$ [9], which we considered in the previous section.

The Bäcklund transformations represented by (58)-(64) correspond to second iterations, with appropriate choices of signs, of (65)-(69). The Bäcklund transformations represented by (65)-(69) can be found in [6] (see also [7]), and correspond to a composition of what appear to be the only previously known auto-Bäcklund transformations for $P_{V}$ in the case $\delta \neq 0[3,4,9]$. These known Bäcklund transformations, as well as this composition, are derived in our approach by consideration of the ODE satisfied by $\rho(x)$, as we now indicate.

We eliminate $Q$ between the Riccati equation

$$
\rho^{\prime}=\delta \rho^{2}+\left(2 \delta Q(x)-\frac{\delta+\gamma+\delta^{2} x}{\delta x}\right) \rho+\frac{1}{2 \delta x}(\delta(\alpha+\beta-1)-\gamma)(2 Q-1)
$$

and the solution (65) for $\rho$ and obtain a second order ODE for $\rho$. The change of variable

$$
\rho=\frac{(\delta(\alpha+\beta-1)-\gamma)}{2 \delta^{2} x}(w-1)
$$

then yields

$$
w^{\prime \prime}=\left(\frac{1}{2 w}+\frac{1}{w-1}\right) w^{\prime 2}-\frac{w^{\prime}}{x}+\frac{(w-1)^{2}}{2 x^{2}}\left(A^{2} w-\frac{B^{2}}{w}\right)+\frac{C w}{x}-\frac{D^{2} w(w+1)}{2(w-1)},
$$

where 


$$
\begin{array}{ll}
A^{2}=\frac{(\delta(\alpha+\beta)-\delta-\gamma)^{2}}{4 \delta^{2}}, & C=\delta(\beta-\alpha), \\
B^{2}=\frac{(\delta(\alpha+\beta)-\delta+\gamma)^{2}}{4 \delta^{2}}, & D^{2}=\delta^{2} .
\end{array}
$$

Equation (73) is a copy of $P_{V}$ in canonical form, and so we see that we have deduced a further Bäcklund transformation for $P_{V}$. That is, equation (73) is related to (39) by

$$
m=\frac{(\delta(\alpha+\beta)-\delta-\gamma) w^{2}-(\delta(\alpha+\beta)-\delta+\gamma)+2 \gamma w+2 \delta^{2} x w+2 \delta x w^{\prime}}{4 \delta^{2} x w},
$$

and then to our original copy of $P_{V}$ in canonical form (37) via $y=m /(m-1)$, with parameters related by (74), (75). Equation (73) is related to our equation in $Q$ by a Bäcklund transformation equivalent, up to choices of sign, to (74)-(76).

The class of Bäcklund transformations (74)-(76) was given in $[\mathbf{3}, \mathbf{4}, \mathbf{9}]$. We therefore see that, for appropriate choices of signs, the Bäcklund transformations represented by (65)-(69) can be written as compositions of these known Bäcklund transformations. The class of Bäcklund transformations (74)-(76) give rise to Bäcklund transformations of $P_{V}$ that are fundamental: the derivation given here is analogous to that given in [1] of the fundamental Bäcklund transformations $\hat{y}$ and $\tilde{y}$ of the fourth Painlevé equation (which were also obtained when considering the ODE satisfied by $\rho(x)$ ).

Finally, we note that for (58)-(64), consideration of the ODE in $\rho(x)$ leads to a Bäcklund transformation between $P_{I I I}$ and an ODE of second order and second degree.

4. Conclusions. We have applied our approach to obtaining Bäcklund transformations, which is based on mappings preserving natural subsets of movable poles, to $P_{I I I}$ and $P_{V}$. For $P_{I I I}$ we have recovered the four fundamental Bäcklund transformations for the case $\gamma \delta \neq 0$, as well as what appears to be a new general formulation of the second iteration of these or of a further Bäcklund transformation which exists in the case $\gamma \delta=0$. For $P_{V}$ our approach allows us to recover what appear to be all known nontrivial Bäcklund transformations. A crucial point in obtaining such general results for $P_{I I I}$ and $P_{V}$ is our allowing $\tau$ to depend not only on $x$ but also on $Q(x)$.

Acknowledgements. The research of PRG and AP was supported in part by the DGICYT under contract PB98-0262. NJ's research was supported by the Australian Research Council under Grant \#A69803721 and Fellowship \#F69700172. AP thanks the Ministry of Education and Culture of Spain for a post-doctoral fellowship.

5. Appendix. In this appendix we briefly compare the approach outlined here with the standard Painlevé truncation. We take as an example the third Painlevé equation $P_{I I I}$.

Seeking a solution of (2) in the form of a so-called truncated Painlevé expansion, we obtain 


$$
y=\frac{\varphi^{\prime}}{\gamma \varphi}-\frac{1}{2 \gamma}\left(\frac{\varphi^{\prime \prime}}{\varphi^{\prime}}+\frac{\alpha+\gamma}{\gamma x}\right),
$$

together with an equation of the form $A \varphi^{-1}+B=0$, for some $A, B$.

The truncated expansion (77) can be compared with the results obtained in Section 2 by linearising the Riccati equation (8), (15) by setting $\rho=\varphi^{\prime} /(\gamma \varphi)$, which then leads to

$$
Q=-\frac{1}{2 \gamma-\frac{(a-\alpha) \varphi}{x \varphi^{\prime}}}\left(\frac{\varphi^{\prime \prime}}{\varphi^{\prime}}+\frac{\alpha+\gamma}{\gamma x}\right)
$$

and thus (3) becomes

$$
y=\frac{\varphi^{\prime}}{\gamma \varphi}-\frac{1}{2 \gamma-\frac{(a-\alpha) \varphi}{x \varphi^{\prime}}}\left(\frac{\varphi^{\prime \prime}}{\varphi^{\prime}}+\frac{\alpha+\gamma}{\gamma x}\right) .
$$

This is the same as the truncated Painleve expansion (77) only in the case $a=\alpha$, and thus we see that the only results obtainable using (77) will be those for this restricted case. That is, we can only obtain the identity or restricted cases of the Bäcklund transformations presented in Section 3. Deriving these results requires applying the method presented in $[\mathbf{1 0}, \mathbf{1 1}]$ rather than a standard truncation approach.

Similar remarks hold for $P_{I V}$ and $P_{V}$, or in general when the function $\tau \neq 0$.

\section{REFERENCES}

1. P. R. Gordoa, N. Joshi and A. Pickering, Mapping preserving locations of movable poles: a new extension of the truncation method to ordinary differential equations, Nonlinearity 12 (1999), 955-968.

2. J. Weiss, The Painlevé property for partial differential equations II: Bäcklund transformations, Lax pairs, and the Schwarzian derivative, J. Math. Phys. 24 (1983), 1405-1413.

3. V. I. Gromak, Solutions of Painlevé's fifth equation, Differential Equations 12 (1976), $519-521$.

4. A. S. Fokas and M. J. Ablowitz, On a unified approach to transformations and elementary solutions of Painlevé equations, J. Math. Phys. 23 (1982), 2033-2042.

5. K. Okamoto, Studies on the Painlevé equations IV. Third Painlevé equation $P_{I I I}$, Funkcial Ekvac. 30 (1987), 305-322.

6. K. Okamoto, Studies on the Painlevé equations II. Fifth Painlevé equation $P_{V}$, Japan J. Math. 13 (1987), 47-76.

7. J. Schiff, Bäcklund transformations of MKdV and Painlevé equations, Nonlinearity 7 (1994), 305-312.

8. A. E. Milne, P. A. Clarkson and A. P. Bassom, Bäcklund transformations and solution hierarchies for the third Painlevé equation, Stud. Appl. Math. 98 (1997), 139-194.

9. V. I. Gromak, Bäcklund transformations of Painlevé equations and their applications, in The Painlevé Property. One Century Later, ed. R. Conte (Springer-Verlag, 1999), 687-734.

10. P. A. Clarkson, N. Joshi and A. Pickering, Bäcklund transformations for the second Painlevé hierarchy: a modified truncation approach, Inverse Problems 15 (1999), 175-187.

11. N. Joshi and A. Pickering, Bäcklund transformations for similarity reductions of the modified Sawada-Kotera/Kaup-Kupershmidt hierarchy, preprint (1999). 